\title{
Insights from Screening a Racially and Ethnically Diverse Population for Chronic Kidney Disease
}

\author{
Linda L. Wong ${ }^{a}$ Kamyar Kalantar-Zadeh ${ }^{c}$ Victoria Page ${ }^{b}$ \\ Glen Hayashida ${ }^{\mathrm{b}}$ Amy S. You ${ }^{\mathrm{c}}$ Connie M. Rhee ${ }^{\mathrm{c}}$ \\ ${ }^{a}$ Department of Surgery, University of Hawaii John A. Burns School of Medicine, and \\ ${ }^{\mathrm{b}}$ National Kidney Foundation - Hawaii Chapter, Honolulu, Hawaii; ' ${ }^{\mathrm{D}}$ ivision of Nephrology and \\ Hypertension, University of California Irvine School of Medicine, Orange, CA, USA
}

\section{Keywords}

Hawaii · Asian · Pacific Islander · Minorities ·

Chronic kidney disease

\begin{abstract}
Background: The value of chronic kidney disease (CKD) screening in the general population remains unclear but may be beneficial in populations with high disease prevalence. We examined risk factors for albuminuria among participants in a state-wide CKD screening program in Hawaii. Methods: The National Kidney Foundation of Hawaii Kidney Early Detection Screening (NKFH-KEDS) program held 19 CKD screening events from 2006 to 2012. Participants rotated through 5 stations during which sociodemographic, blood glucose, urine albumin-to-creatinine ratio (ACR), and spot urine albumin data were collected. Multivariate logistic regression analyses (adjusted for age, sex, race/ethnicity, body mass index [BMI]) were used to identify clinical predictors of abnormal ACR ( $\geq 30 \mu \mathrm{g} / \mathrm{mg})$ and abnormal spot urine albumin (>20 mg/L) levels. Results: Among 1,190 NKFH-KEDS participants who met eligibility criteria, 13 and $49 \%$ had abnormal ACR and urine albumin levels, respectively. In multivariate logistic regression analyses, participants of older age (>65 years), Asian and Pacific Islander race/ethnicity, BMI $\geq 30 \mathrm{~kg} / \mathrm{m}^{2}$, and with hypertension had higher risk of abnor-
\end{abstract}

mal ACR. Being of older age; Asian, Pacific Islander, and Mixed race/ethnicity; and having diabetes was associated with higher risk of abnormal urine albumin levels in adjusted analyses. Conclusions: NKFH-KEDS participants of older age; Asian and Pacific Islander race/ethnicity; and with obesity, hypertension, and diabetes had higher risk of kidney damage defined by elevated ACR and urine albumin levels. Further studies are needed to determine whether targeted screening programs can result in timely identification of CKD and implementation of interventions that reduce cardiovascular disease, death, and progression to end-stage renal disease.

(c) 2017 S. Karger AG, Basel

\section{Introduction}

In the United States (US), more than 20 million adults, or 1 in 10 US residents suffer from chronic kidney disease (CKD) [1]. As of 2013, there were 661,648 prevalent cases of end-stage renal disease (ESRD) in the US, among whom 466,607 were receiving dialysis [2]. There were 86,965 dialysis patients as well as an additional 14,541 pre-dialysis CKD patients awaiting kidney transplantation in 2013 with 48,311 active candidates on the transplant list, yet only 17,600 patients received a transplant,

\section{KARGER}

(c) 2017 S. Karger AG, Basel

E-Mail karger@karger.com

www.karger.com/ajn
Connie M. Rhee, MD, MSc

Division of Nephrology and Hypertension

University of California Irvine School of Medicine

101 The City Drive South, City Tower, Suite 400, Orange, CA 92868 (USA)

E-Mail crhee1@uci.edu 
yielding a waiting list that was 2.7 -fold higher than the supply of donor kidneys [2]. Furthermore, the 2013 costs of ESRD patients was $\$ 30.9$ billion, accounting for $7.1 \%$ of the overall Medicare paid claims costs in the fee-forservice system [2]. Although kidney transplantation is more cost-effective than maintaining a patient on dialysis, there is a dearth of viable organs to meet the demands of eligible ESRD patients. Over time, patients have endured longer waiting time intervals for kidney transplantation, and the ever-widening gap between the supply and demand of kidneys has been difficult to close. As efforts to increase organ donation may only minimally impact the insufficient organ supply, the most effective way to address this problem will be to reduce the demands for kidney transplantation. Ultimately, recognition of CKD in its earlier stages and preventing progression toward ESRD will have the greatest impact on addressing the kidney supply-demand mismatch.

To this end, developing screening programs in populations with a high prevalence of kidney disease risk factors using noninvasive, inexpensive, and reliable methods has the potential to identify patients with CKD who may be at risk of developing ESRD. Furthermore, given that markers of early kidney disease (e.g., albuminuria, proteinuria) have demonstrated potent associations with cardiovascular disease and death [3], timely identification and targeted interventions (i.e., administration of angiotensin-converting enzyme inhibitors, blood pressure control, weight loss) may ameliorate the exceedingly high mortality of this population. There is a paucity of data demonstrating the benefit of a one-time screening test for $\mathrm{CKD}$, and it has yet to be shown that screening can improve the outcomes of CKD patients. As such, the American College of Physicians does not support screening for CKD in asymptomatic adults, although the evidence for this recommendation is weak [4].

However, it has been suggested that the effectiveness of a CKD screening program may vary according to the racial/ethnic background of the source population. Hawaii has the highest prevalence of Asian and Pacific Islanders among whom there is a greater burden of CKD $[5,6]$. Indeed, the state of Hawaii has demonstrated a higher incidence and prevalence of ESRD patients receiving dialysis compared to the national average [7]. Although there has been considerable progress in our understanding of the risk factors for and sequelae of CKD among African-Americans and Latin Americans [8, 9], there have been few published studies of CKD in the Asian and Pacific Islander populations to date $[6,10,11]$. For example, while multiple traditional risk factors for
CKD are highly prevalent in Hawaii [6, 7], little is known about the specific risk factors for early CKD (i.e., elevated albuminuria and proteinuria levels) in this under-recognized "high-risk" population. To address this knowledge gap, in 2005, the National Kidney Foundation of Hawaii (NKFH) developed the Kidney Early Detection Screening (KEDS) program with the objective of raising CKD awareness and promoting early kidney disease screening among its residents across the state of Hawaii. In the following study, we aimed to (1) describe the implementation and operational activities of the NKFH-KEDS screening program model, and to (2) examine rich participant-level data collected from a racially and ethnically diverse group of participants during NKFH-KEDS health screening events [10]. In terms of the latter objective, we sought to examine characteristics associated with specific markers of early kidney damage, namely abnormal urine albumin-to-creatinine ratio (ACR) and spot urine albumin levels, among participants in the KEDS program.

\section{Methods}

Source Cohort

This is a cross-sectional study of participants in Wave 1 (20062009) of the NKFH-KEDS program. Data examined in this study were collected over the course of 19 KEDS health screening events ("KEDS events") conducted at the Hawaii State Capitol grounds and shopping malls, community colleges, community health centers, hospitals, and community centers throughout the state of Hawaii including the islands of Maui, Oahu, Kauai, and the Big Island. These KEDS sites were selected in collaboration with community partners, and site requirements included adequate space availability, affordability, accessibility, and convenience to the public, as well the ability to meet the screening events' logistical requirements. The study was approved by the Institutional Review Boards and Committee on Human Studies at the University of Hawaii and the University of California Irvine Medical Center.

\section{KEDS Events Procedures}

Each KEDS event was staffed by approximately 25-45 volunteers affiliated with NKFH. Volunteers consisted of healthcare professionals (physicians, nurses, pharmacists, dietitians, and physical therapists), trainees in the healthcare field, and the lay community who were required to undergo formalized training prior to participation. Initial training was conducted in small groups, and in 2010, a standardized video module was developed to facilitate the training of new volunteers (www.youtube.com/ kedsorientation). A second orientation was conducted immediately prior to each KEDS event and included a review of (1) the purpose of the screening events and program procedures, (2) paperwork and documentation, (3) interviewing techniques, (4) equipment protocols for blood testing, (5) physical measurements, (6) urinalysis, and (7) screening follow-up recommendations. Participation was completely voluntary, and those who enrolled were not paid. Upon enrollment, trained volunteers explained the 

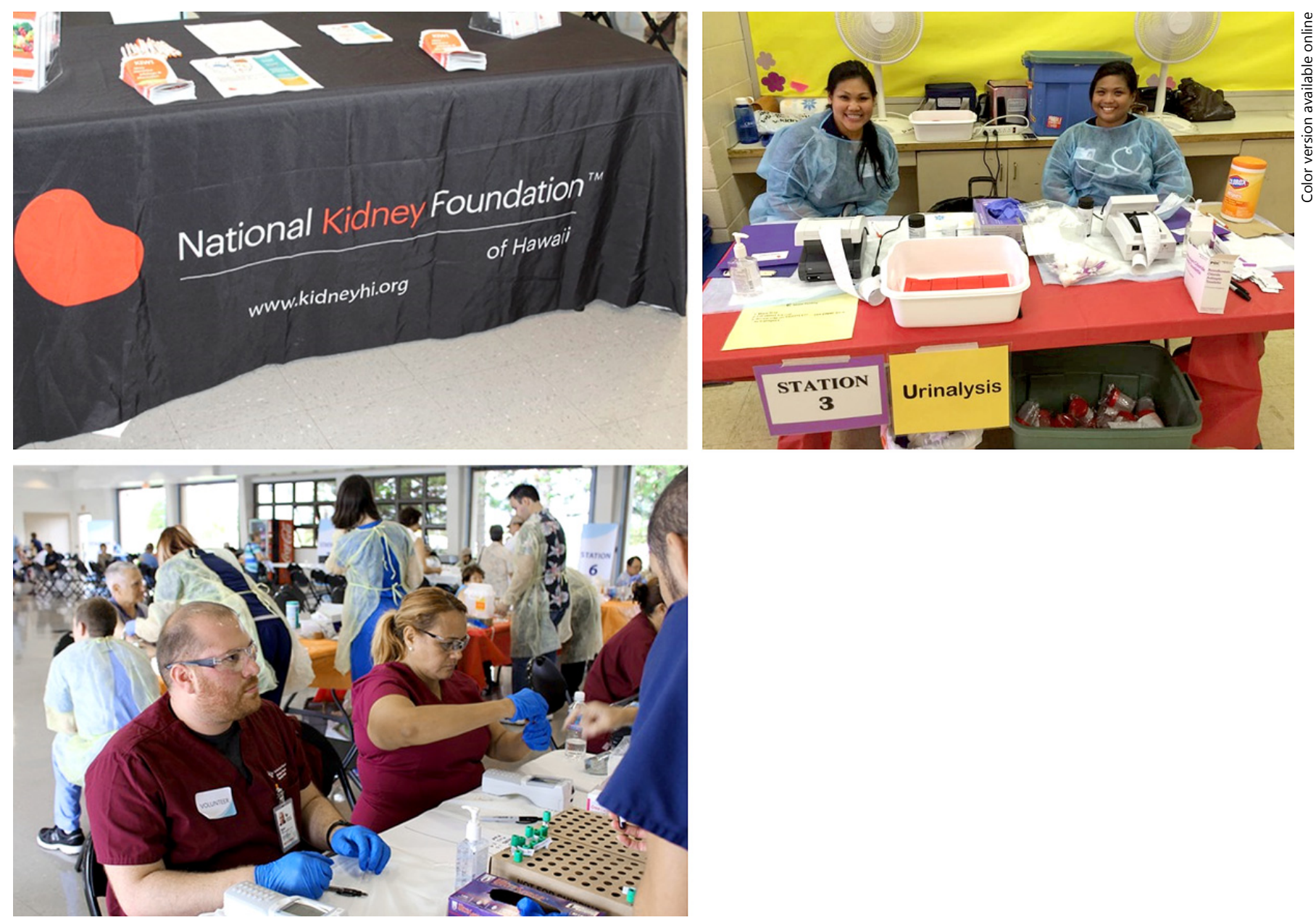

Fig. 1. Photos from National Kidney Foundation of Hawaii Kidney Early Detection Screening Program events.

rationale for the program, obtained informed consent, and provided a description of the following 5 stations screening participants would rotate through (Fig. 1):

1. Station 1 (registration): participants registered for the event and completed a health assessment form that collected information on sociodemographics (age, sex, self-reported race/ethnicity), comorbidities (hypertension, diabetes, elevated cholesterol, cardiovascular disease, kidney disease), smoking history, family history of comorbidities (hypertension, diabetes, elevated cholesterol, cardiovascular disease, kidney disease), and use of medications for conditions such as hypertension, cholester$\mathrm{ol}$, and kidney disease. Volunteers were available to assist participants with visual impairments or language barriers.

2. Station 2 (physical measurements): volunteers who were students or healthcare professionals performed blood pressure, height, and weight measurements, the latter of which were used to calculate body mass index (BMI).

3. Station 3 (urinalysis): volunteers provided participants with a specimen cup and instructions on how to obtain a "clean-catch" urine sample. Specimens were processed utilizing either a Clinitek 50 or Clinitek Status Analyzer. Bayer/Siemens Diagnostics Microalbumin Reagent test strips were utilized.
4. Station 4 (blood draw): venous or capillary blood specimens were collected by phlebotomy professionals. Venous blood specimens were transported via couriers to local laboratories for processing. For capillary blood specimens, Accu-Check Aviva blood glucose meters and test strips by Roche were used.

5. Station 5 (exit interview): healthcare professionals who included physicians, physician assistants, nurse practitioners, and registered nurses conducted exit interviews with each of the participants, which involved reviewing their screening test results and providing general recommendations and education regarding CKD risk factors. Participants with abnormal, noncritical test results were advised to promptly follow-up with their primary care providers. Results from the venous blood specimen collection were mailed to the participants' homes 7-10 days after screening.

Fliers and radio/newspaper advertisements were primarily used to notify the community of the KEDS events. For screenings held in larger communities, interested participants were allowed to walk-in without an appointment or pre-register by phone. KEDS events held in smaller rural communities required word-ofmouth communication by leaders in the community, placement of banners, and other grassroots tactics, given that radio/newspaper 
advertisements were not as successful in disseminating screening event information. Community members who were interested in undergoing screening during the KEDS events were enrolled in the program irrespective of health insurance, health conditions, sex, and race/ethnicity, and each KEDS event had the capacity to accommodate up to 150 participants.

\section{Statistical Analysis}

Descriptive statistics were estimated to examine KEDS participants' sociodemographics. Participants' race/ethnicity was categorized as White, Asian, Pacific Islander, Black, American Indian, and Mixed race/ethnicity. Participants who had at least 50\% Pacific Islander race/ethnicity were categorized as "Pacific Islander." Those who listed 2 or more race/ethnicities were categorized as "Mixed," unless they indicated theywere atleast $50 \%$ Pacific Islander in background. Given the very small number of Black $(n=6)$ and American Indian $(n=6)$ participants, participants of these racial/ ethnic backgrounds were included in sensitivity analyses but excluded from the primary analyses.

We first examined the association between the participants' sociodemographics and clinical characteristics with 2 co-primary outcome measures which included (1) urine ACR and (2) spot urine albumin levels. Based on Kidney Disease Improving Global Outcome definitions, ACR was reported as normal (ACR $<30 \mu \mathrm{g} / \mathrm{mg}$ ), presence of microalbuminuria (ACR $30-300 \mu \mathrm{g} / \mathrm{mg}$ ), and presence of macroalbuminuria (ACR $>300 \mu \mathrm{g} / \mathrm{mg}$ ), and spot urine albumin levels were reported as normal (urine albumin $<20 \mathrm{mg} / \mathrm{L}$ ) and abnormal (urine albumin $\geq 20 \mathrm{mg} / \mathrm{L}$ ) [12-14]. Given the sparse numbers of participants with non-normal ACR levels, we conducted univariate logistic regression analyses to examine characteristics associated with an ACR dichotomized as normal (ACR $<30 \mu \mathrm{g} / \mathrm{mg}$ ) vs. abnormal (ACR $\geq 30 \mu \mathrm{g} / \mathrm{mg}$ ). Multivariate logistic regression models were also used to determine characteristics independently associated with abnormal ACR and spot urine albumin levels, which included the following covariates: age (dichotomized as $>65$ vs. $\leq 65$ years), sex, race/ethnicity, and BMI (dichotomized as $\geq 30$ vs. $<30 \mathrm{~kg} / \mathrm{m}^{2}$ ). Complete case analysis was used for the multivariate regression models.

We then compared clinical characteristics among participants with normal vs. abnormal ACR levels using $t$ tests, chi-square, and Fisher exact tests, depending upon data type. Analogous analyses were conducted for normal vs. abnormal spot urine albumin levels. Data were analyzed using Excel, SPSS version 22 (IBM, Armonk, NY, USA), SAS version 9.4 (SAS Institute Inc., Cary, NC, USA), and Stata version 13.1 (Stata Corporation, College Station, TX, USA).

\section{Results}

\section{Description of Study Population}

There were 1,277 participants in the NKFH-KEDS program among whom the mean \pm SD age was $55.7 \pm$ 16.4 years and $62 \%$ were female. The racial/ethnic distribution of the cohort was as follows: Asians (43\%), Whites (23\%), Pacific Islanders (13\%), Mixed race/ethnicity $(11 \%)$, Black $(<1 \%)$, American Indian $(<1 \%)$, and unknown race/ethnicity (10\%). The mean \pm SD BMI was
$28.0 \pm 6.5 \mathrm{~kg} / \mathrm{m}^{2}$, with $29 \%$ and $14 \%$ of participants meeting criteria for obesity $\left(\mathrm{BMI} \geq 30 \mathrm{~kg} / \mathrm{m}^{2}\right)$ and morbid obesity (BMI $\left.\geq 35 \mathrm{~kg} / \mathrm{m}^{2}\right)$, respectively.

Baseline characteristics of participants with ACR data examined in primary analyses $(n=1,178)$ overall and stratified according to ACR level are shown in Table 1. In the overall cohort, the following comorbidity prevalences were observed: hypertension (41\%), elevated cholesterol (30\%), diabetes (26\%), cardiovascular disease (5\%), and kidney disease (3\%). Approximately $7 \%$ of participants reported active smoking. A large proportion of the participants reported having a family history of diabetes (42\%), heart disease $(31 \%)$, and kidney disease (11\%), while $<1 \%$ of participants reported having a family history of hypertension. Compared to participants with normal ACR levels $(<30 \mu \mathrm{g} / \mathrm{mg})$, those with abnormal ACR levels ( $\geq 30 \mu \mathrm{g} / \mathrm{mg}$ ) were less likely to be White and more likely to be Asian or Pacific Islander; were more likely to have hypertension, diabetes, and elevated cholesterol; and were more likely to have a family history of diabetes.

\section{Urine ACR Levels}

ACR data were available among 1,190 participants, among whom the distribution of values was as follows: normal (ACR $<30 \mu \mathrm{g} / \mathrm{mg} ; n=1,030$ ), presence of microalbuminuria (ACR $30-300 \mu \mathrm{g} / \mathrm{mg} ; n=147$ ), and presence of macroalbuminuria (ACR $>300 \mu \mathrm{g} / \mathrm{mg} ; n=13$; Fig. 2a).

In primary analyses (overall cohort $n=1,178$ ), we found that being of older age ( $>65$ years), Asian and Pacific Islander race/ethnicity, and having obesity (BMI $\geq 30 \mathrm{~kg} / \mathrm{m}^{2}$ ), hypertension, diabetes, and elevated cholesterol were associated with higher risk of having abnormal ACR levels using univariate logistic regression (online suppl. Table 1; for all online suppl. material, see www. karger.com/doi/10.1159/000455389). Following adjustment for case-mix characteristics in multivariate logistic regression analyses, participants of older age ( $>65$ years), Asian and Pacific Islander race/ethnicity, obese BMI, and with underlying hypertension persisted in having a higher risk of abnormal ACR (Table 2 ). In sensitivity analyses that included Black $(n=6)$ and American Indian $(n=6)$ participants (overall cohort $n=1,190$ ), we observed the same pattern of associations (data not shown).

\section{Spot Urine Albumin Levels}

Spot urine albumin data were available among 1,190 participants, among whom the distribution of values was as follows: normal (urine albumin $<20 \mathrm{mg} / \mathrm{L} ; n=606$ ) and abnormal (urine albumin $\geq 20 \mathrm{mg} / \mathrm{L} ; n=584$; Fig. $2 \mathrm{~b}$ ). 


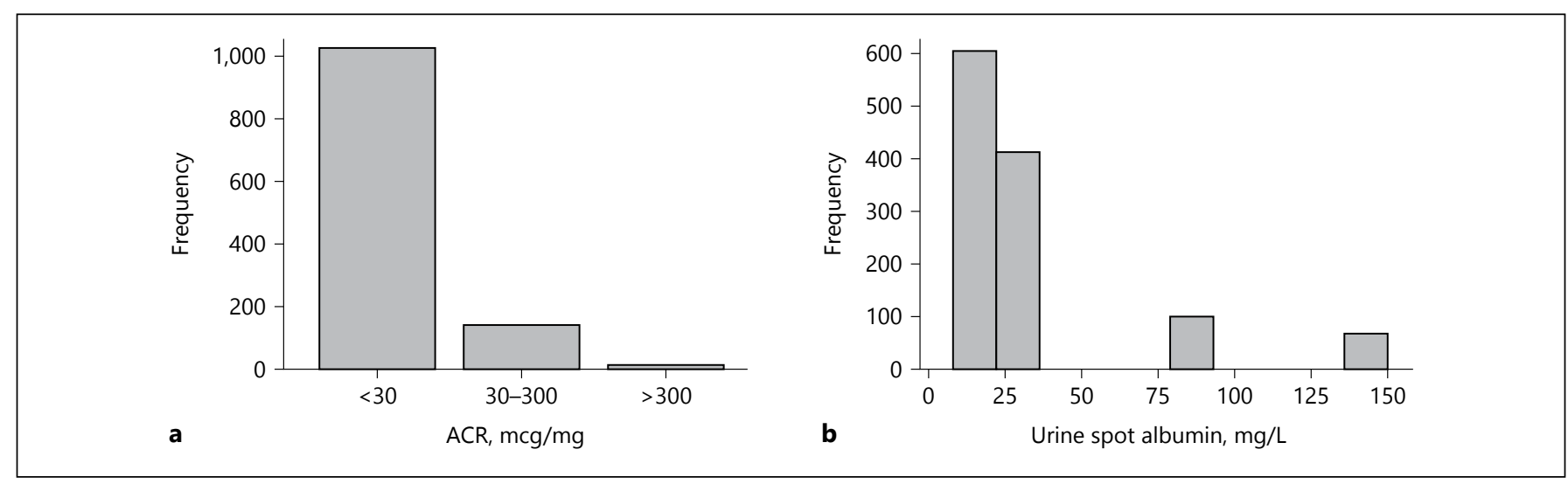

Fig. 2. Distribution of urine albumin-to-creatinine (a) and spot urine albumin (b) levels among 1,190 KEDS participants.

Table 1. Baseline characteristics in the overall cohort and according to ACR level

\begin{tabular}{|c|c|c|c|c|}
\hline \multirow[t]{2}{*}{ Characteristic } & \multicolumn{3}{|l|}{$\mathrm{ACR}, \mu \mathrm{g} / \mathrm{mg}$} & \multirow[t]{2}{*}{$p$ value } \\
\hline & overall cohort & $\mathrm{ACR}<30$ & $\mathrm{ACR} \geq 30$ & \\
\hline Number (\%) & $1,178(100)$ & $1,019(87)$ & $159(13)$ & N/A \\
\hline Age, years, mean $\pm S D$ & $55.9 \pm 16.3$ & $55.6 \pm 16.2$ & $57.9 \pm 17.0$ & 0.116 \\
\hline Female, \% & 61 & 62 & 58 & 0.381 \\
\hline Race, \% & & & & 0.009 \\
\hline White & 25 & 27 & 17 & \\
\hline Asian & 48 & 48 & 50 & \\
\hline Pacific Islander & 15 & 14 & 22 & \\
\hline Mixed race/ethnicity & 12 & 12 & 11 & \\
\hline $\mathrm{BMI}, \mathrm{kg} / \mathrm{m}^{2}$, mean $\pm \mathrm{SD}$ & $28.1 \pm 6.5$ & $28.0 \pm 6.3$ & $28.8 \pm 7.4$ & 0.138 \\
\hline HTN, \% & 41 & 38 & 56 & $<0.001$ \\
\hline Diabetes, \% & 26 & 25 & 33 & 0.027 \\
\hline Elevated cholesterol, \% & 30 & 29 & 40 & 0.007 \\
\hline Cardiovascular disease, $\%$ & 5 & 5 & 6 & 0.892 \\
\hline Kidney disease, $\%$ & 3 & 3 & 5 & 0.067 \\
\hline Smoking, \% & 7 & 7 & 6 & 0.600 \\
\hline Family history of HTN, \% & $<1$ & $<1$ & $<1$ & 0.500 \\
\hline Family history of diabetes, $\%$ & 42 & 41 & 45 & 0.358 \\
\hline Family history of cardiovascular disease, $\%$ & 31 & 31 & 32 & 0.991 \\
\hline Family history of kidney disease, $\%$ & 11 & 11 & 11 & 0.843 \\
\hline
\end{tabular}

BMI, body mass index; HTN, hypertension; ACR, albumin-to-creatinine ratio.

$p$ value calculated by $t$ test or Kruskal-Wallis test.

In primary analyses (overall cohort $n=1,178$ ), we found that being of Pacific Islander race/ethnicity, obese BMI $\left(\geq 30 \mathrm{~kg} / \mathrm{m}^{2}\right)$, and having diabetes was associated with higher risk of having abnormal urine albumin levels using univariate logistic regression (online suppl. Table 2). Following adjustment for case-mix characteristics in multivariate logistic regression analyses, participants of older age ( $>65$ years); Asian, Pacific Islander, and Mixed race/ethnicity; and with underlying diabetes had a higher risk of abnormal urine albumin levels (Table 3 ). In sensitivity analyses that included Black $(n=6)$ and American Indian $(n=6)$ participants (overall cohort $n=1,190$ ), we observed the same pattern of associations (data not shown).

In crude analyses examining urine albumin levels according to sociodemographic and clinical characteristics 
Table 2. Characteristics associated with an abnormal urine ACR level (ACR $\geq 30 \mu \mathrm{g} / \mathrm{mg}$ ) using multivariate logistic regression models

\begin{tabular}{llll}
\hline Characteristic & OR & $95 \%$ CI & $p$ value \\
\hline Age $>65$ years & $\mathbf{1 . 5 9}$ & $\mathbf{1 . 0 7 - 2 . 3 5}$ & $\mathbf{0 . 0 2 1}$ \\
Sex, female & 0.83 & $0.57-1.21$ & 0.331 \\
Asian* & $\mathbf{1 . 8 7}$ & $\mathbf{1 . 1 1 - 3 . 1 4}$ & $\mathbf{0 . 0 1 9}$ \\
Pacific Islander* & $\mathbf{2 . 6 6}$ & $\mathbf{1 . 4 4 - 4 . 9 3}$ & $\mathbf{0 . 0 0 2}$ \\
Mixed race/ethnicity* & 1.58 & $0.76-3.28$ & 0.219 \\
BMI $\geq 30 \mathrm{~kg} / \mathrm{m}^{2}$ & $\mathbf{1 . 5 4}$ & $\mathbf{1 . 0 1 - 2 . 3 3}$ & $\mathbf{0 . 0 4 3}$ \\
HTN & $\mathbf{1 . 5 9}$ & $\mathbf{1 . 0 9}-\mathbf{2 . 3 3}$ & $\mathbf{0 . 0 1 6}$ \\
Diabetes & 1.21 & $0.81-1.82$ & 0.354 \\
Elevated cholesterol & 1.33 & $0.90-1.96$ & 0.149 \\
Cardiovascular disease & 0.89 & $0.39-2.05$ & 0.791 \\
Kidney disease & 1.32 & $0.48-3.66$ & 0.595 \\
Smoking & 0.80 & $0.37-1.74$ & 0.576 \\
Family history of HTN & 4.72 & $0.46-48.45$ & 0.192 \\
Family history diabetes & 1.08 & $0.74-1.57$ & 0.695 \\
Family history of cardiovascular disease & 1.04 & $0.69-1.56$ & 0.859 \\
Family history of kidney disease & 0.89 & $0.49-1.59$ & 0.685 \\
\hline
\end{tabular}

* Reference group: Whites.

BMI, body mass index; HTN, hypertension; ACR, albumin-to-creatinine ratio.

Analyses adjusted for age (dichotomized as $\leq 65$ [reference] vs. $>65$ years), sex, race/ethnicity (White [reference], Asian, Pacific Islander, Mixed), BMI ( $<30$ [reference] vs. $\left.\geq 30 \mathrm{~kg} / \mathrm{m}^{2}\right)$.

Bold estimates indicate significant predictors of abnormal urine ACR level.

Table 3. Characteristics associated with an abnormal urine microalbumin level $(>20 \mathrm{mg} / \mathrm{L})$ using multivariate logistic regression models

\begin{tabular}{llll}
\hline Characteristic & OR & $95 \%$ CI & $p$ value \\
\hline Age $>65$ years & $\mathbf{1 . 3 8}$ & $\mathbf{1 . 0 3 - 1 . 8 3}$ & $\mathbf{0 . 0 2 8}$ \\
Sex, female & 0.80 & $0.62-1.04$ & 0.092 \\
Asian* & $\mathbf{1 . 5 0}$ & $\mathbf{1 . 0 9 - 2 . 0 6}$ & $\mathbf{0 . 0 1 2}$ \\
Pacific Islander* & $\mathbf{1 . 5 8}$ & $\mathbf{1 . 0 3}-\mathbf{2 . 4 3}$ & $\mathbf{0 . 0 3 8}$ \\
Mixed race/ethnicity* & $\mathbf{2 . 3 2}$ & $\mathbf{1 . 4 4 - 3 . 7 2}$ & $\mathbf{0 . 0 0 1}$ \\
BMI $\geq 30 \mathrm{~kg} / \mathrm{m}^{2}$ & 1.31 & $0.97-1.77$ & 0.075 \\
HTN & 1.03 & $0.79-1.35$ & 0.824 \\
Diabetes & $\mathbf{1 . 4 2}$ & $\mathbf{1 . 0 6}-\mathbf{1 . 9 1}$ & $\mathbf{0 . 0 2 1}$ \\
Elevated cholesterol & 1.03 & $0.78-1.37$ & 0.836 \\
Cardiovascular disease & 0.76 & $0.44-1.34$ & 0.344 \\
Kidney disease & 1.02 & $0.47-2.23$ & 0.956 \\
Smoking & 0.75 & $0.45-1.25$ & 0.268 \\
Family history of HTN & 1.83 & $0.25-13.31$ & 0.553 \\
Family history diabetes & 1.16 & $0.89-1.51$ & 0.271 \\
Family history of cardiovascular disease & 1.05 & $0.79-1.39$ & 0.752 \\
Family history of kidney disease & 1.39 & $0.93-2.08$ & 0.111 \\
\hline
\end{tabular}

* Reference group: Whites.

BMI, body mass index; HTN, hypertension.

Analyses adjusted for age (dichotomized as $\leq 65$ [reference] vs. $>65$ years), sex, race/ethnicity (White [reference], Asian, Pacific Islander, Mixed), BMI ( $<30$ [reference] vs. $\geq 30 \mathrm{~kg} / \mathrm{m}^{2}$ ).

Bold estimates indicate significant predictors of abnormal urine microalbumin level. 
(overall cohort $n=1,178$ ), we observed that participants who were age $>65$ years; with a BMI $\geq 30 \mathrm{~kg} / \mathrm{m}^{2}$; with underlying hypertension, diabetes, or high cholesterol; or Pacific Islander race/ethnicity had higher mean urine albumin levels compared with those who did not have these characteristics (online suppl. Table 3 ). In contrast, White participants had lower mean urine albumin levels compared to non-White participants.

\section{Discussion}

By leveraging rich participant-level data collected from the KEDS screening outreach program, we have had the opportunity to (1) describe NKFH's procotolized approach in implementing screening events among a racially and ethnically diverse community; (2) report the benefits of these far-reaching screening efforts in identifying early stages of CKD among high-risk communities across the state of Hawaii; and (3) examine the dominant factors associated with early kidney damage (i.e., elevated ACR and albuminuria) in this study population.

In this study, we observed that $13 \%$ and $49 \%$ of community participants who attended KEDS health screening events were found to have abnormal ACR and urine albumin levels, respectively. Those who were identified to have risk factors or abnormal tests at screening were encouraged to follow-up with their primary care providers and were enrolled in a follow-up educational program (Kidney Interactive Workshop and Information). We additionally observed that a large proportion of the cohort had obesity and morbid obesity (29\% and $13 \%$, respectively). Our crude analyses demonstrated that participants of older age; Asian and Pacific Islander race/ethnicity; and with underlying obesity, hypertension, diabetes, and elevated cholesterol had higher risk of abnormal ACR levels. After accounting for differences in case-mix characteristics, logistic regression analyses demonstrated that older age, Asian and Pacific Islander race/ethnicity, obese BMI, and underlying hypertension were independently associated with higher risk of abnormal ACR. Similarly, adjusted analyses demonstrated that older age, Asian and Pacific Islander and Mixed race/ethnicity, and diabetes were independently associated with higher risk of abnormal urine albumin levels.

Examination of data collected by the NKFH-KEDS program provides the unique opportunity to understand the characteristics of a racially and ethnically diverse group of CKD health screening participants across the state of Hawaii, which has a high prevalence of Asians,
Pacific Islanders, and Mixed Racial/Ethnic groups [15]. Hawaii has a higher incidence and prevalence rate of patients with ESRD on dialysis compared to the national average; among these patients, over $60 \%$ have diabetes $[6$, 7]. With the intention of focusing upon community members at "high-risk" for developing CKD, the unrestricted KEDS screening cohort $(n=1,277)$ was comprised of racially/ethnically diverse participants who, compared to the broader Hawaii population [16], had a lower prevalence of White participants ( $23 \%$ vs. $44 \%$, respectively) but a similarly high prevalence of Asians ( $43 \%$ vs. $56 \%$ ), Pacific Islanders (13\% vs. $11 \%$ ), and those of Mixed race/ethnicity (11\% vs. $23 \%)$. Compared to the state-wide population [16], the unrestricted KEDS cohort had a higher prevalence of diabetes ( $9 \%$ vs. $26 \%$ ), higher prevalence of hypertension (32\% vs. $40 \%$ ), and a similar prevalence of obesity ( $23 \%$ vs. $29 \%$ ).

These findings are an important addition to scarce existing literature on risk factors for CKD in Hawaii. While native Hawaiians, Pacific Islanders, and Asians have a higher risk of developing CKD compared to White counterparts $[17,18]$, there is limited understanding of the underlying risk factors specific to this population. A large body of evidence has shown that native Hawaiians and Pacific Islanders in both the CKD and non-CKD populations have a higher prevalence of diabetes, hypertension, and obesity compared to other racial/ethnic groups $[5,6]$. While diabetes and hypertension have been recognized as major risk factors for CKD in the native Hawaiian/Pacific Islander population, our study demonstrates that obesity is independently associated with higher risk of kidney damage, manifested as abnormal ACR levels, in this population. A large body of evidence has shown that obesity leads to the development of incident CKD via direct mechanisms including increased glomerular hyperfiltration and alterations in renal hemodynamics, inflammatory cytokines, adipokine production, and transforming growth factor-beta production [19]. In addition, obesity may indirectly lead to CKD development and progression vis-à-vis increased hypertension, diabetes, and atherosclerosis. Recent data have shown that interventions targeted at reducing obesity may reverse or retard CKD progression. For example, two large case series have shown that bariatric surgery among severely/morbidly obese patients resulted in improvement in estimated glomerular filtration rates and/or abnormal ACR levels [20, 21]. Notably, we observed that there was a disproportionate prevalence of CKD risk factors (e.g., diabetes, hypertension) relative to the proportion of participants with high ACR levels. Given that the study was conducted amongst com- 
munity members who volunteered to participate in the NKFH-KEDS screening events, it is possible that these participants may have been healthier compared to the general population (i.e., despite having a high prevalence of CKD risk factors, this cohort may have had better access to care, adherence to lifestyle and pharmacotherapeutic interventions, and more well-controlled diabetes and hypertension) and that the scope of early CKD in Hawaii may be greater than observed in this cohort. Thus, implementing screening events in communities with a high prevalence of obesity, as well as interventions targeting weight management, may have important implications upon the kidney health of these populations.

By identifying modifiable factors associated with abnormal levels of ACR and urine albumin (e.g., obesity, hypertension), our findings may provide insights into potential interventions that can ameliorate the substantially high burden of cardiovascular mortality (i.e., second leading cause of death) among high-risk populations in Hawaii [22]. Epidemiologic data have shown that incrementally higher levels of albuminuria are associated with increasing risk of all-cause and cardiovascular mortality [3]. Given the high prevalence of abnormal ACR and urine albumin levels observed in the KEDS cohort (i.e., 13 and $49 \%$, respectively), screening events targeted at highrisk communities may additionally lead to early identification and interventions toward cardiovascular risk factors with potential downstream effects upon reducing cardiovascular disease and death in this population.

Several limitations of the study bear mention. First, while KEDS events were open to all community members, participants who chose to attend health fairs and screenings may have been inherently different compared to the general population, resulting in potential selection bias. Second, the study is also limited by reliability upon par- ticipants' self-report of medical history and family history of comorbidities. Third, we lacked data on the proportion of participants who completed the entire screening process (as opposed to partial completion), as well as the duration of time required to complete screening activities. A fourth limitation of the study includes its collection of ACR and urine albumin data at a single point in time. As there may be variations in specimen collection and machine calibration, one-time measurements of these values may not capture participants' typical or longitudinal values. Lastly, data linkage to subsequent medical care and long-term follow-up information were not available.

Despite these limitations, this study represents a firsttime examination of a very large and racially/ethnically diverse cohort of participants who underwent CKD screening throughout the state of Hawaii. While there may be limited evidence to support the screening of the general population for $\mathrm{CKD}$, targeting a high-risk population (i.e., high prevalence of Asian and Pacific Islanders, obesity, diabetes, and hypertension) may have potential value. Further studies are needed to determine whether screening programs targeted at Hawaii residents with a high prevalence of CKD risk factors can reduce the overall burden of ESRD in this population.

\section{Grant Support}

The authors are supported by the research grants from the NIH/ NIDDK including K23-DK102903 (C.M.R.) and K24-DK091419 (K.K.-Z.).

\section{Disclosure Statement}

None of the authors have disclosures to report.

\section{References}

1 Centers for Disease Control and Prevention (CDC): National Chronic Kidney Disease Fact Sheet: General Information and National Estimates on Chronic Kidney Disease in the United States, 2014. Atlanta, US Department of Health and Human Services, Centers for Disease Control and Prevention, 2014.

2 US Renal Data System: USRDS 2015 Annual Data Report: Atlas of End-Stage Renal Disease in the United States. Bethesda, USRDS, 2015.

3 van der Velde M, Matsushita K, Coresh J, Astor BC, Woodward M, Levey A, de Jong P, Gansevoort RT; Chronic Kidney Disease
Prognosis Consortium, van der Velde M, Matsushita K, Coresh J, Astor BC, Woodward M, Levey AS, de Jong PE, Gansevoort RT, Levey A, El-Nahas M, Eckardt KU, Kasiske BL, Ninomiya T, Chalmers J, Macmahon S, Tonelli M, Hemmelgarn B, Sacks F, Curhan G, Collins AJ, Li S, Chen SC, Hawaii Cohort KP, Lee BJ, Ishani A, Neaton J, Svendsen K, Mann JF, Yusuf S, Teo KK, Gao P, Nelson RG, Knowler WC, Bilo HJ, Joosten H, Kleefstra N, Groenier KH, Auguste P, Veldhuis K, Wang Y, Camarata L, Thomas B, Manley T: Lower estimated glomerular filtration rate and higher albuminuria are associated with all-cause and cardiovascular mortality. A collaborative meta-analysis of high-risk population cohorts. Kidney Int 2011;79:1341-1352.

4 Qaseem A, Hopkins RH Jr, Sweet DE, Starkey M, Shekelle P: Screening, monitoring, and treatment of stage 1 to 3 chronic kidney disease: a clinical practice guideline from the American College of Physicians. Ann Intern Med 2013;159:835-847.

5 Hall YN, Sugihara JG, Go AS, Chertow GM: Differential mortality and transplantation rates among Asians and Pacific Islanders with ESRD. J Am Soc Nephrol 2005;16:37113720 . 
6 Mau MK, West M, Sugihara J, Kamaka M, Mikami J, Cheng SF: Renal disease disparities in Asian and Pacific-based populations in Hawai'i. J Natl Med Assoc 2003;95:955963.

7 Hawaii Diabetes Report 2004. http://health. hawaii.gov/diabetes/files/2013/10/2004diabe tesreport.pdf (accessed November 13, 2016).

8 Sankare IC, Bross R, Brown AF, Del Pino HE, Jones LF, Morris DM, Porter C, Lucas-Wright A, Vargas R, Forge N, Norris KC, Kahn KL: Strategies to build trust and Recruit African American and Latino community residents for health research: a cohort study. Clin Transl Sci 2015;8:412-420.

9 Tareen N, Zadshir A, Martins D, Pan D, Nicholas S, Norris K: Chronic kidney disease in African American and Mexican American populations. Kidney Int Suppl 2005;97:S137S140.

10 Kataoka-Yahiro MR, Wong KA, Tamashiro J, Page V, Ching J, Li D: Evaluation of the National Kidney Foundation of Hawai'i's Kidney Early Detection Screening program. Hawaii J Med Public Health 2012;71:186192.

11 Lee B, Turley M, Meng D, Zhou Y, Garrido T, Lau A, Radler L: Effects of proactive population-based nephrologist oversight on pro- gression of chronic kidney disease: a retrospective control analysis. BMC Health Serv Res 2012;12:252.

12 de Zeeuw D, Parving HH, Henning RH: Microalbuminuria as an early marker for cardiovascular disease. J Am Soc Nephrol 2006;17: 2100-2105.

13 Jafar TH, Chaturvedi N, Hatcher J, Levey AS: Use of albumin creatinine ratio and urine albumin concentration as a screening test for albuminuria in an Indo-Asian population. Nephrol Dial Transplant 2007;22:21942200.

14 Levey AS, Eckardt KU, Tsukamoto Y, Levin A, Coresh J, Rossert J, De Zeeuw D, Hostetter TH, Lameire N, Eknoyan G: Definition and classification of chronic kidney disease: a position statement from Kidney Disease: Improving Global Outcomes (KDIGO). Kidney Int 2005;67:2089-2100.

15 US Census Bureau. http://quickfacts. census.gov/qfd/states/15000.html (accessed December 26, 2015).

16 Department of Business, Economic Development, and Tourism. Census. Hawaii Population Characteristics 2015. http://files.hawaii. gov/dbedt/census/popestimate/2015_ county_char_hi_file/Pop_char_hi_2015 final.pdf (accessed November 13, 2016).
17 Hall YN, Hsu CY: New insights into the epidemiology of chronic kidney disease in US Asians and Pacific Islanders. Curr Opin Nephrol Hypertens 2006;15:264-269.

18 Hall YN, Hsu CY, Iribarren C, Darbinian J, McCulloch CE, Go AS: The conundrum of increased burden of end-stage renal disease in Asians. Kidney Int 2005;68:2310-2316.

19 Rhee CM, Ahmadi SF, Kalantar-Zadeh K: The dual roles of obesity in chronic kidney disease: a review of the current literature. Curr Opin Nephrol Hypertens 2016;25:208-216.

20 Amor A, Jimenez A, Moize V, Ibarzabal A, Flores L, Lacy AM, Vidal J: Weight loss independently predicts urinary albumin excretion normalization in morbidly obese type 2 diabetic patients undergoing bariatric surgery. Surg Endosc 2013;27:2046-2051.

21 Hou CC, Shyu RS, Lee WJ, Ser KH, Lee YC Chen SC: Improved renal function 12 months after bariatric surgery. Surg Obes Relat Dis 2013;9:202-206.

22 American Heart Association/American Stroke Association Hawaii State Fact Sheet. Leading Causes of Death in Hawaii in 2010. https:// www.heart.org/idc/groups/heart public/@wcm/@adv/documents/downloadable/ucm_307165.pdf(accessed November 13, 2016). 